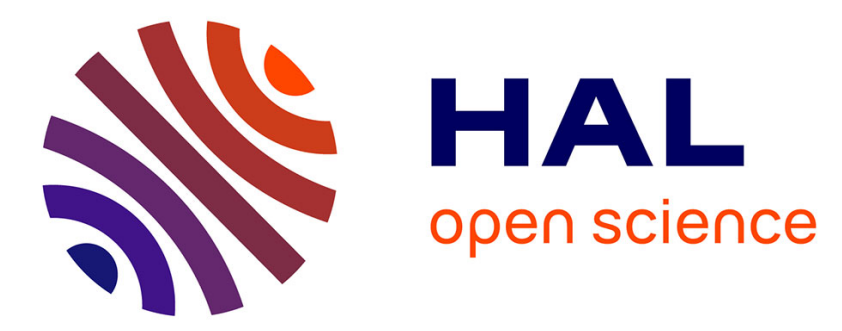

\title{
The prognostic value of the neutrophil-to-lymphocyte ratio in critically ill cirrhotic patients
}

\author{
Mikhael Giabicani, Emmanuel Weiss, Gerald Chanques, Caroline Lemaitre, \\ Audrey de Jong, Steven Grangé, Richard Moreau, Gaël Piton, Catherine \\ Paugam-Burtz, Samir Jaber, et al.
}

\section{To cite this version:}

Mikhael Giabicani, Emmanuel Weiss, Gerald Chanques, Caroline Lemaitre, Audrey de Jong, et al.. The prognostic value of the neutrophil-to-lymphocyte ratio in critically ill cirrhotic patients. European Journal of Gastroenterology \& Hepatology, 2021, Publish Ahead of Print, 10.1097/MEG.0000000000002063 . hal-03117608

\section{HAL Id: hal-03117608 \\ https://hal.science/hal-03117608}

Submitted on 21 Jan 2021

HAL is a multi-disciplinary open access archive for the deposit and dissemination of scientific research documents, whether they are published or not. The documents may come from teaching and research institutions in France or abroad, or from public or private research centers.
L'archive ouverte pluridisciplinaire $\mathbf{H A L}$, est destinée au dépôt et à la diffusion de documents scientifiques de niveau recherche, publiés ou non, émanant des établissements d'enseignement et de recherche français ou étrangers, des laboratoires publics ou privés. 


\section{The prognostic value of the neutrophil-to- lymphocyte ratio in critically ill cirrhotic patients}

Mikhael Giabicani ${ }^{a, b, \star}$, Emmanuel Weiss ${ }^{a, c, \star}$, Gerald Chanques ${ }^{d}$, Caroline Lemaitre ${ }^{b}$, Audrey De Jong ${ }^{d}$, Steven Grangéb, Richard Moreau ${ }^{\mathrm{c}, \mathrm{e}}$, Gaël Piton ${ }^{\mathrm{f}}$, Catherine Paugam-Burtz ${ }^{\mathrm{a}, \mathrm{c}}$, Samir Jaber ${ }^{\mathrm{d}}$ and Fabienne Tamion ${ }^{\mathrm{b}, \mathrm{g}}$

Background Hospital death rates following ICU admission of cirrhotic patients remain high. Identifying patients at high risk of mortality after few days of aggressive management is imperative for providing adequate interventions. Herein, we aimed to evaluate the prognostic value of the neutrophil-to-lymphocyte ratio (NLR) combined with usual organ failure scores in the outcome prediction of cirrhotic patients hospitalized more than 3 days in ICU.

Methods We conducted a retrospective bicentric study in two cohorts of cirrhotic patients hospitalized more than 3 days in French university hospital ICUs. At admission and day 3, we calculated several clinico-biological scores grading liver disease and organ failure severity and calculated the NLR. The primary outcome was 28-day mortality.

Results The test cohort included 116 patients. At day 28, 43 (37.1\%) patients had died. Variations of MELD score ( $\triangle M E L D)$,

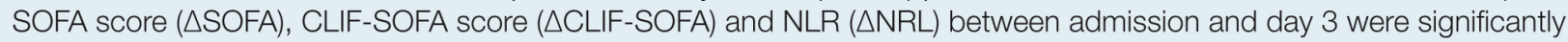
associated with 28-day mortality in univariate analysis. When included in bivariate analysis $\triangle N L R$ remained a significant predictor of 28-day mortality independently of these severity scores. Kaplan-Meier curves and statistics using reclassification methods showed a better 28-day mortality risk prediction using $\triangle N R L$ in association with $\triangle S O F A$ in comparison to $\triangle S O F A$ alone. These results were confirmed in an external validation cohort, including 101 critically ill cirrhotic patients. Conclusions $\triangle N L R$ is an independent predictor of mortality in the critically ill cirrhotic patients' population who requires intensive care supportive treatment and should be used in association with $\triangle$ SOFA as a prognostic biomarker.

\section{Introduction}

Hospital death rates following ICU admission of cirrhotic patients are lower than previously reported and have improved over a few years $[1,2]$. However, it remains high and ranges from 44 to $86 \%[3,4]$. In this context, it is difficult to predict which patients will benefit from ICU admission, especially in nontransplant centers. The use of scoring systems has been proposed to inform clinical

European Journal of Gastroenterology \& Hepatology 2020, XXX:00-00

Keywords: cirrhosis, critically ill cirrhotic patient, ICU, mortality, neutrophil-to-lymphocyte ratio, sequential organ failure assessment

aDepartment of Anesthesiology and Critical Care, APHP, Beaujon Hospital, Clichy, 'bedical Intensive Care Unit, Rouen University Hospital, Rouen, CUMR_S 1149 Centre for research on Inflammation - Inserm/Université de Paris, Paris, dAnesthesiology and Intensive Care, Anesthesia and Critical Care Department B, Saint Eloi Teaching Hospital, PhyMedExp, University of Montpellier, INSERM U1046, CNRS, UMR 9214, Montpellier, 'épeartement Hospitalo-Universitaire UNITY, Service d'Hépatologie, APHP, Hôpital Beaujon, Clichy, ${ }^{\mathrm{f}}$ Medical Intensive Care Unit, University Hospital of Besancon, Besançon and 9Normandie University, UNIROUEN, INSERM U1096 EnVI, Rouen, France

Correspondence to Mikhael Giabicani, MD, Department of Anesthesiology and Critical Care, Beaujon Hospital, 100, Boulevard du Général Leclerc, 92110 Clichy, France

Tel: +33140875911; e-mail: mikhael.giabicani@aphp.fr

*Mikhael Giabicani and Emmanuel Weiss contributed equally to the writing of the manuscript. decision-making, but in this population, the sequential organ failure assessment score (SOFA), the acute physiology and chronic health evaluation II score (APACHE II), and the chronic liver failure-sequential organ failure assessment score (CLIF-SOFA) are not sufficiently informative for predicting mortality upon ICU admission $[1,4]$. This lack of early and accurate predictor justifies initial permissive ICU admission policies and deferred prognostic assessment after 3 days of aggressive management. At day 3 , some patients are no longer hospitalized in ICU, because already deceased or discharged. However, most of the patients are still hospitalized, and intensive care escalation has to be discussed given the expected poor course of the disease $[5,6]$. To date, this deferred decision is solely based on organ failure scores whose prognostic ability in this setting has been evaluated by a few studies only $[4,5]$. Nevertheless, identifying patients that are at high risk of mortality after day 3 and accurately anticipating outcomes is imperative for providing adequate interventions.

The neutrophil-to-lymphocyte ratio (NLR) is a biological marker available from daily blood routine test that has been shown to be associated with outcomes in unselected critically ill patients [7] and in patients with stable liver cirrhosis $[8,9]$. It may be a promising prognostic marker for the clinical decision-making process to decide if life-sustaining therapies should be maintained in critically ill cirrhotic patients.

The aim of our study was to evaluate whether the NLR may help in choosing the optimal treatment strategies for 


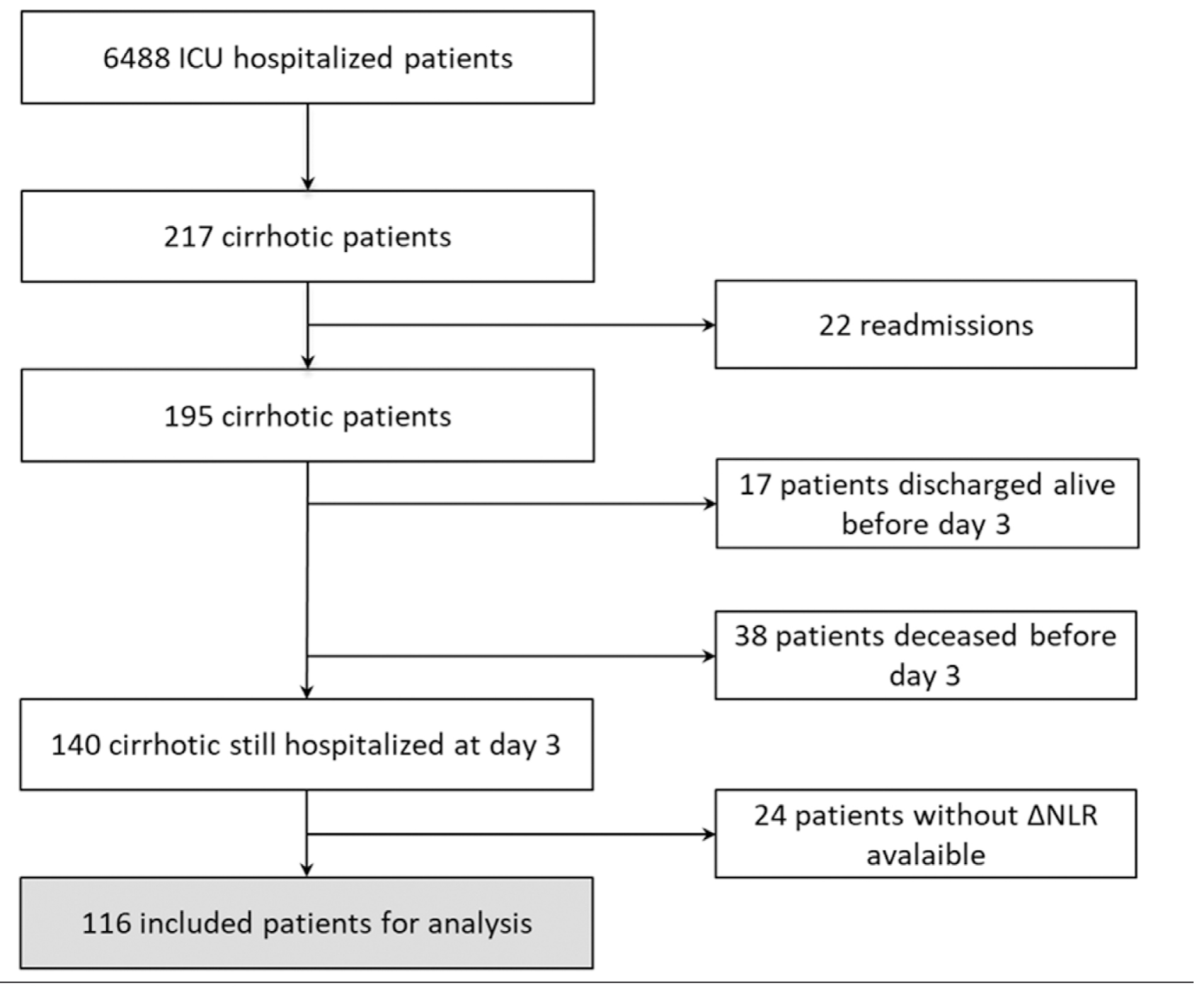

Fig. 1. Flow chart of included patients.

critically ill cirrhotic patients still hospitalized in ICU after 3 days of full organ support by improving the prediction of their outcome.

\section{Methods \\ Patients}

All consecutive cirrhotic patients admitted in two independent ICUs were retrospectively reviewed and those still alive at day 3 were included in the study. Exclusion criteria included ICU length of stay $<3$ days, age $<18$ years, complete blood count data unavailable, rehospitalization in the same ICU (only the first hospitalization was considered). Cirrhosis diagnosis was based either on liver biopsy or on combination of clinical (jaundice, ascites, hepatic encephalopathy), laboratory (hyperbilirubinaemia, hypoalbuminemia, prolonged prothrombin time, thrombocytopaenia) or radiological (ultrasound, computed tomography or MRI) findings.

This study was approved by the ethical committee of the French Society of Anesthesia and Intensive Care Medicine (NIRB 00010254-2019-033).

Two different cohorts were defined a test cohort (Medical ICU, Rouen University Hospital, France) in which statistical analyzes were first performed and an external validation cohort (St Eloi ICU, Montpellier University Hospital, France) to confirm the results obtained in the test cohort. The inclusion period ranged between January 2010 and December 2016 for the test cohort and between January 2013 and June 2017 for the validation cohort.

\section{Data collection}

Because recent evidence suggests that a dynamic assessment of ICU prognostic scores between admission and day 3 may be more accurate than fixed-day values [10], this approach was used to address the aim of the study. For each patient, clinical and biological variables were collected at admission and at day 3. Clinical data included demographic characteristics, ICU admission diagnosis and the main clinical events for hospitalization (uncontrolled ascites, variceal bleeding, hepatic encephalopathy). Biological data included biochemical, hematological and bacteriological data.

NLR was computed based as a ratio of neutrophil/lymphocyte values, which are recorded in the complete blood count.

Several clinico-biological scores grading liver disease (Child-Pugh score, MELD score) and organ failure 
severity [IGS II, SOFA score, CLIF-SOFA score, CLIF-C acute decompensation, CLIF-C ACLF, acute-on-chronic liver failure (ACLF) grade] were recorded. Patients were also classified as ACLF, pre-ACLF and acute decompensation according to the European Association for the Study of the Liver-CLIF Consortium definition [11]. The changes in selected severity scores ( $\triangle$ MELD, $\triangle$ SOFA, $\triangle$ CLIFSOFA) and in NLR ( $\triangle$ NLR) were calculated as the difference between values at day 3 and at admission.

\section{Outcome variables}

The primary outcome was 28-day mortality. Secondary outcomes were ICU mortality, in-hospital mortality, 90-day mortality and secondary infections (defined as a second infectious episode for patients admitted for sepsis and as nosocomial infections occurring during ICU stay for the others). The vital status was confirmed by the review of the electronic medical records of the hospitals.

\section{Statistical analyzes}

Data for continuous variables were expressed as mean \pm 1 SEM or median with interquartile range and were compared using Wilcoxon's rank test. Qualitative variables were displayed as numbers and percentages and were compared using Fisher's exact test.

Univariate regression analyzes using Cox proportional hazards models were conducted to investigate whether changes in organ failure scores $(\triangle \mathrm{SOFA}, \triangle \mathrm{CLIF}-S O F A$ and $\triangle$ MELD scores) and in NLR ( $\triangle$ NLR) were associated with 28-day mortality. $\triangle$ NLR was then introduced into bivariate Cox proportional hazards models with organ failure scores achieving a $P$ value $<0.05$. Data for patients who survived were censored at the date of hospital release or of the last follow-up visit.

The incremental contribution of $\triangle N L R$ to the mortality prediction on the top of organ failure scores was assessed using different methods. Firstly, survival curves according to the trends in $\triangle$ SOFA alone and $\triangle$ SOFA combined with $\triangle$ NLR were built using the Kaplan-Meier method and compared by log-rank test. Secondly, the continuous net reclassification improvement (cNRI) index, which is used to measure the ability of a new model (with the new biomarker, in this case $\triangle \mathrm{NLR}$ ) to reclassify a highrisk individual as higher risk and a low-risk individual as lower risk $[7,12,13]$ was calculated. A positive continuous NRI means a more accurate risk prediction using the new model while negative continuous NRI indicates a worse risk prediction. Moreover, continuous NRI values above 0.6 are classically considered strong [14].

All reported tests of the data are two-sided with a significance level of $5 \%$, and confidence interval (CIs) are reported as two-sided $95 \%$. Statistical analyzes were performed using SPSS 22.0.0 (SPSS Inc., Chicago, Illinois, USA) and the R PredictABEL package.

\section{Results}

\section{Patients' characteristics in the test cohort}

Among the 6488 patients admitted in the ICU of Rouen University Hospital during the study period, 217 (3.3\%) had cirrhosis and were screened. After exclusion of 101 patients who meet the exclusion criteria, 116 patients were included in the test cohort (Fig. 1). Characteristics of the patients are displayed in Table 1 . The main cause of liver disease was alcohol-related disease and median MELD score was $26(20-31)$. Ninety-nine patients $(85.3 \%)$ were classified as ACLF [median CLIF-C ACLF $=58(52-63)$ ], 5 patients $(4.3 \%)$ as pre-ACLF and 12 patients $(10.3 \%)$ as acute decompensation [median CLIF-C acute decompensation $=60$ (51-66) and 54 (48-58), respectively]. ACLF patients were classified as ACLF grade $1(n=12,10.3 \%)$, grade $2(n=35,30.2 \%)$ or grade $3(n=52,44.8 \%)$ according to the number of organ failures. Main reasons for ICU admission were sepsis or septic shock and gastrointestinal

Table 1. Characteristics of the patients in the test cohort and the validation cohort

\begin{tabular}{|c|c|c|c|}
\hline Characteristics & $\begin{array}{l}\text { Test cohort } \\
(n=116)\end{array}$ & $\begin{array}{l}\text { Validation cohort } \\
\qquad(n=101)\end{array}$ & $P$ \\
\hline Sex (F/M) & $33 / 83(1 / 2.5)$ & $35 / 66(1 / 1.9)$ & 0.33 \\
\hline Age & $57(50-64]$ & $58(51-65)$ & 0.56 \\
\hline Child score & $9(7-11]$ & $10(8-10)$ & 0.04 \\
\hline \multicolumn{4}{|l|}{ Etiologies of cirrhosis } \\
\hline Alcohol & $108(93.1 \%)$ & $76(75.2 \%)$ & $<0.0$ \\
\hline HBV & $2(1.7 \%)$ & $2(2 \%)$ & 0.89 \\
\hline $\mathrm{HCV}$ & $6(5.2 \%)$ & $13(12.9 \%)$ & 0.04 \\
\hline $\mathrm{NASH}$ & $9(7.8 \%)$ & $12(11.9 \%)$ & 0.31 \\
\hline Other & $4(3.4 \%)$ & $12(11.9 \%)$ & 0.02 \\
\hline \multicolumn{4}{|l|}{ Reason for admission } \\
\hline Septic shock or sepsis & $70(60.3 \%)$ & $55(54.5 \%)$ & 0.38 \\
\hline Gastrointestinal bleeding & $19(16.4 \%)$ & $26(25.7 \%)$ & 0.09 \\
\hline Acute & & $2(2 \%)$ & 0.21 \\
\hline Hepa & & & 0.1 \\
\hline Other & $19(16.4 \%)$ & 12 & 0.34 \\
\hline \multicolumn{4}{|c|}{ Organ failure and severity scores at admission } \\
\hline Acute renal failure & $81(69.8 \%)$ & $\%)$ & 0.11 \\
\hline KDIG & & & $<0.001$ \\
\hline dacement therapy & $10(8.6$ & $4(3.9 \%)$ & 0.16 \\
\hline Mechanical ventilation & $72(51.4 \%)$ & $37(36,6 \%)$ & 0.023 \\
\hline pathy & 42( & $\%)$ & 0.05 \\
\hline IGS? & $51(3$ & 40 & $<0.001$ \\
\hline SOFA & $11(7-13)$ & $8(6-11)$ & $<0.01$ \\
\hline MELD score at DO & $26(20-31)$ & $19(14-28)$ & $<0.00$ \\
\hline CLIF & $13(\varepsilon$ & $9(7-11)$ & $<0.0$ \\
\hline ACLF grade & & $1(0-2)$ & 508 \\
\hline \multicolumn{4}{|c|}{ Biological parameters at admission } \\
\hline Leucocytes at DO (G/L) & $13.2(7.9-18.5)$ & $8.4(4.9-14.7)$ & $<0.00$ \\
\hline Neutr & 11.1 & 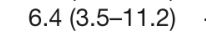 & $<0.00$ \\
\hline Lym & & $0.9(0$. & 0.35 \\
\hline NLR a & $10.9(6-17.5$ & $6.8(4.4-12)$ & $<0$. \\
\hline Total bilirubin at $\mathrm{DO}(\mu \mathrm{mol} / \mathrm{L})$ & $60.5(27.0-144.5)$ & $83(34-156)$ & 0.23 \\
\hline INR at DO & $2.0(1.6-2.8)$ & $1.8(1.5-2.3)$ & 0.04 \\
\hline \multicolumn{4}{|c|}{ Organ failure and severity scores at day 3} \\
\hline SOFA score at D3 & $9(6-15)$ & $6(4$ & $<0.0$ \\
\hline MELD s & $26(19-32)$ & $19(14-26)$ & $<0.00$ \\
\hline CLIF-SOFA score at D3 & $10(7-16)$ & $8(6-10)$ & $<0.00$ \\
\hline \multicolumn{4}{|l|}{ Biological parameters at admission } \\
\hline Leucocytes at D3 (G/L) & $10.7(7$ & $7.9(4.9$ & $<0$. \\
\hline Neutrophils at D3 (G/L) & $8.6(5.8-14.6)$ & $5.8(3.3-10.0)$ & $<0.00$ \\
\hline Lymphocytes at D3 (G/L) & $0.8(0.6-1.2)$ & $0.9(0.5-1.3)$ & 0.74 \\
\hline NLR at D3 & $10.8(6$ & $6.3(4.2-10.5)$ & $<0.00$ \\
\hline $\mathrm{D} 3(\mu \mathrm{mol} / \mathrm{L}$ & $87(32-205)$ & $82(37-212)$ & 0.81 \\
\hline INR & $6-2.8)$ & $1.9(1.5-$ & \\
\hline \multicolumn{4}{|c|}{ Dynamic variations of NLR and severity scores } \\
\hline$\Delta \mathrm{NLR}$ & $-0.3(-6.7-5.2]$ & $0.03(-3.9$ to 3.1$)$ & 0.62 \\
\hline$\triangle S O F A$ & $0(-3$ to 3$)$ & $-2(-4$ to 0$)$ & $<0.01$ \\
\hline$\triangle$ CLIF-SOFA & $-1(-3$ to 2$)$ & $-1(-2$ to 0$)$ & 0.77 \\
\hline$\triangle M E L D$ & $0(-4$ to 3$)$ & $-1(-4$ to 2$)$ & 0.34 \\
\hline
\end{tabular}

ACLF, acute-on-chronic liver failure; CLIF-SOFA, chronic liver failure-sequential organ failure assessment; HBV, hepatitis B virus; HVC, hepatitis $\mathrm{C}$ virus; IGS2, Indice de Gravité Simplifié; INR, international normalized ratio; KDIGO, kidney disease improving global outcomes; MELD, model for end-stage liver disease; NASH, nonalcoholic steatohepatitis; NLR, neutrophil-to-lymphocyte ratio; SOFA, sequential organ failure assessment. 

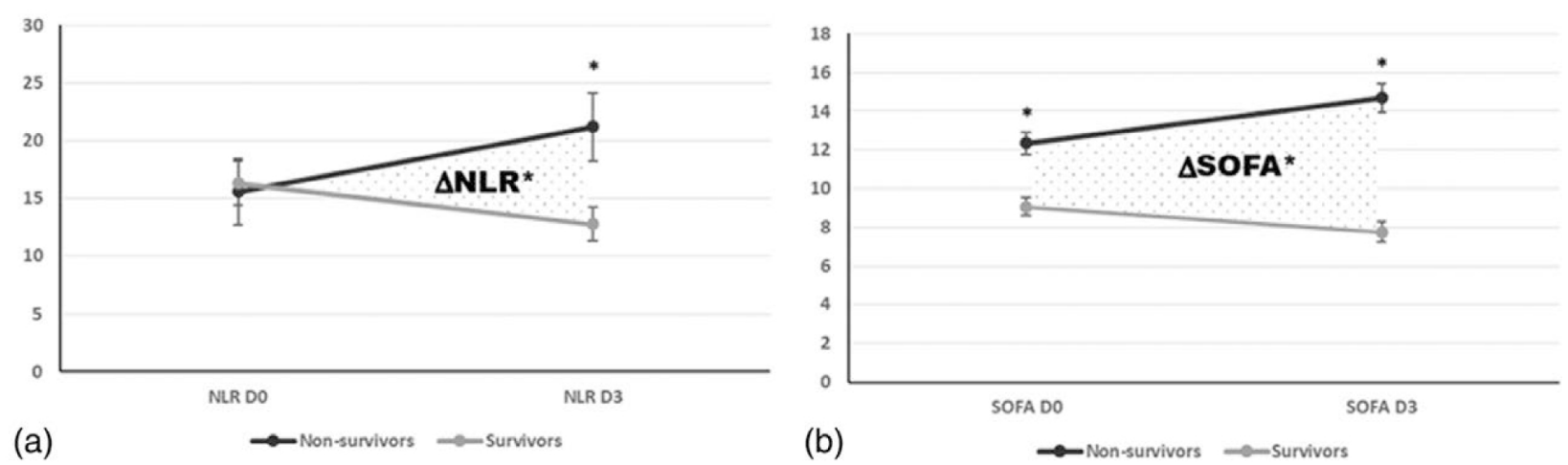

(a) $\rightarrow$ Non-survivors $\rightarrow$ Survivors
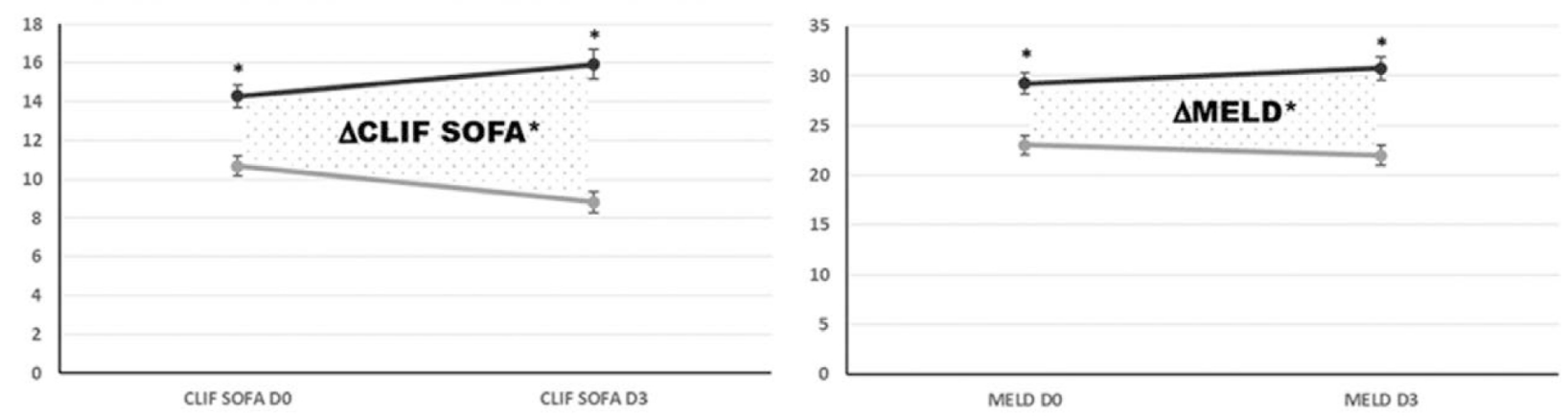

(c)

(d)

Fig. 2. Dynamic variation of NLR (a), SOFA (b), CLIF-SOFA (c) and MELD (d) scores between ICU admission and day 3. Data are presented as mean with SEM. Grey lines and black lines refer to survivors and nonsurvivors, respectively. Dotted areas between the curves indicates the difference in variation of each score ( $\triangle N L R, \triangle S O F A, \triangle C L I F-S O F A, \triangle M E L D)$ between survivors and nonsurvivors. ${ }^{*} P<0.05$ for the difference between survivors and nonsurvivors. CLIF-SOFA, chronic liver failure-sequential organ failure assessment; MELD, model for end-stage liver disease; NLR, neutrophil-to-lymphocyte ratio; SOFA, sequential organ failure assessment.

bleeding. Median ICU and hospital lengths of stay were, respectively, 8 (5-14) and 20 (10-30) days. One patient was transplanted during ICU hospitalization.

\section{Table 1}

\section{Association of neutrophil-to-lymphocyte ratio variation with primary and secondary outcomes}

Patient outcomes are displayed in Supplementary Table 1, Supplement digital content 1, http://links.lww.com/EJGH/ A655. At day 28, 43 (37.1\%) patients had died. $\triangle$ MELD, $\triangle$ SOFA, $\triangle$ CLIF-SOFA and $\triangle$ NRL were significantly higher in nonsurvivors than in survivors. Median values of all of these scores decreased $(\Delta<0)$ between D0 and D3 in survivors, whereas they increased $(\Delta>0)$ in nonsurvivors (Fig. 2). Mortality rates according to the variation of NLR and the severity of the acute decompensation of cirrhosis are displayed in Table 2.

Cox regression analysis for variables associated with 28 -day mortality is shown in Table 3. In univariate analysis, $\triangle$ NLR, $\triangle$ SOFA, $\triangle$ CLIF-SOFA and $\triangle$ MELD scores were associated with primary outcome. When included into bivariate Cox proportional hazards models with $\triangle$ SOFA, $\triangle$ CLIF-SOFA or $\triangle$ MELD, $\triangle$ NLR remained a significant predictor of mortality independently of these severity scores (Table 3). $\triangle$ NLR was also associated, among secondary outcomes, to 90 -day mortality [risk ratio: 1.017; (95\% CI: $1.00-1.03), P=0.01$ ], but not ICU mortality [risk ratio: 1.003; (95\% CI: $0.99-1.02), P=0.68$ ] or secondary infections $(P=0.34$; Supplementary Table 2, Supplement digital content 1, http://links.lww.com/EJGH/ A655).

\section{Tables 2 and 3}

\section{Prognostic value of the combination of neutrophil- to-lymphocyte ratio and sequential organ failure assessment scores}

We used two different approaches to explore the added prognostic value of $\triangle N L R$ to SOFA score, which was kept because as accurate as liver-specific severity scores (CLIF-SOFA and MELD) in our cohort. We first generated Kaplan-Meier curves to evaluate cumulative survival according to both SOFA and NLR variations (Fig. 3). To develop a pragmatic approach for the clinicians, we chose to differentiate patients according to the increase $(\triangle \mathrm{NLR}$ and $\triangle \mathrm{SOFA} \geq 0)$ or a strict decrease $(\triangle \mathrm{NLR}$ and $\triangle$ SOFA $<0$ ) of the NLR and the SOFA score between ICU admission and day 3. As shown in Fig. 3, patients with $\triangle N L R \geq 0$ were at higher risk for 28 -day mortality than the other patients: 68 vs. $48 \%$ if $\triangle \mathrm{SOFA} \geq 0(P=0.05$, Fig. $3 a)$ and 29 vs. $8 \%$ if $\triangle$ SOFA $<0(P=0.04$, Fig. $3 b)$.

As a second approach, we performed reclassification statistics using a continuous cNRI index. We found a cNRI of $0.626(0.266-0.986)$, indicating a strong improvement in the prediction of 28-day mortality using $\triangle$ NLR combined with $\triangle$ SOFA as compared to $\triangle \mathrm{SOFA}$ alone.

\section{Validation cohort}

Characteristics of the 101 patients included in the validation cohort are shown in Table 1. Such as in the test cohort, the diagnosis was based on histological or typical clinico-biological criteria. The etiology of liver cirrhosis was mainly alcoholic $(75.2 \%)$ and the etiology for ICU admission did not significantly differ from the test cohort. 
Table 2. Mortality rates according to the variation of NLR and the severity of the acute decompensation of cirrhosis

\begin{tabular}{|c|c|c|}
\hline ACLF status & $\Delta N L R<0$ & $\Delta \mathrm{NLR} \geq 0$ \\
\hline \multicolumn{3}{|l|}{ No $\operatorname{ACLF}(n=17)$} \\
\hline 28-day mortality (\%) & $0 / 8(0)$ & 2/9 (22.2) \\
\hline \multicolumn{3}{|l|}{ ACLF-1 $(n=12)$} \\
\hline 28-day mortality (\%) & 1/7 (14.2) & $0 / 5(0)$ \\
\hline \multicolumn{3}{|l|}{ ACLF-2 $(n=35)$} \\
\hline 28-day mortality (\%) & 6/22 (27.3) & 7/13 (53.8) \\
\hline \multicolumn{3}{|l|}{ ACLF-3 $(n=52)$} \\
\hline 28-day mortality (\%) & 9/27 (33.3) & $18 / 25(72)$ \\
\hline
\end{tabular}

ACLF, acute-on-chronic liver failure; NLR, neutrophil-to-lymphocyte ratio.

Table 3. Cox regression analysis for variables associated with 28-day mortality in the test and validation cohort

\begin{tabular}{|c|c|c|c|c|c|c|}
\hline \multicolumn{4}{|l|}{ Test cohort } & \multicolumn{3}{|c|}{ Validation cohort } \\
\hline Variables & $\begin{array}{l}\text { Risk } \\
\text { Ratio }\end{array}$ & Cl 95\% & $P$ & $\begin{array}{l}\text { Risk } \\
\text { Ratio }\end{array}$ & $\mathrm{Cl} 95 \%$ & $P$ \\
\hline \multicolumn{7}{|c|}{ Univariate analysis } \\
\hline$\triangle N L R$ & 1,018 & $1.004-1.031$ & $<0.01$ & 1.031 & $1.017-1.045$ & $<0.001$ \\
\hline$\triangle S O F A$ & 1.225 & $1.138-1.318$ & $<0.001$ & 1.144 & $1.014-1.292$ & 0.03 \\
\hline$\triangle$ CLIF-SOFA & 1.207 & $1.121-1.299$ & $<0.001$ & 1.044 & $0.812-1.342$ & 0.74 \\
\hline$\triangle M E L D$ & 1.074 & $1.020-1.130$ & $<0.01$ & 1.012 & $0.911-1.125$ & 0.82 \\
\hline \multicolumn{7}{|l|}{ Bivariate analysis } \\
\hline$\triangle N L R$ & 1.015 & $1.001-1.030$ & 0.03 & 1.029 & $1.015-1.044$ & $<0.001$ \\
\hline$\triangle S O F A$ & 1.229 & $1.137-1.328$ & $<0.001$ & 1.138 & 0.993-1.305 & 0.06 \\
\hline$\triangle N L R$ & 1.017 & $1.003-1.031$ & 0.02 & & & \\
\hline$\triangle$ CLIF-SOFA & 1.214 & $1.124-1.312$ & $<0.001$ & & & \\
\hline$\triangle N L R$ & 1.014 & $1.000-1.029$ & 0.04 & & & \\
\hline$\triangle M E L D$ & 1.065 & $1.010-1.123$ & 0.02 & & & \\
\hline
\end{tabular}

$\mathrm{Cl}$, confidence interval; CLIF-SOFA, chronic liver failure-sequential organ failure assessment; MELD, model for end-stage liver disease; NLR, neutrophil-to-lymphocyte ratio; SOFA, sequential organ failure assessment.

Nevertheless, the patients were less severe and mortality rates were lower (Supplementary Table 1, Supplement digital content 1, bttp://links.lww.com/EJGH/A655). Importantly, the results obtained in the test cohort were confirmed in the validation cohort. Indeed, $\triangle$ NLR predicted mortality independently of $\triangle$ SOFA (Table 3 ) and the risk of death at 28-day was higher in patients with $\triangle N L R \geq 0$ than in those with a negative value (Fig. 3). Finally, reclassification analyzes confirmed the improvement of the mortality risk prediction [CNRI value of 0.624 (0.106-1.141)] using the combination of $\triangle \mathrm{SOFA}$ and $\triangle \mathrm{NLR}$ as compared to $\triangle \mathrm{SOFA}$ alone.

\section{Discussion}

Our study demonstrates the ability of $\triangle$ NLR to predict 28 -day mortality of critically ill cirrhotic patients. We showed that both NLR and ICU or liver-specific severity scores increase during the first 3 days of hospitalization in nonsurvivors, while they decrease in survivors. However, $\triangle \mathrm{NLR}$ was independent of these scores and even added prognostic value to $\triangle \mathrm{SOFA}$, an already known mortality predictor in this population [4]. These results were confirmed in an external validation cohort and then suggest that $\triangle N L R$ may be independently associated with outcomes in cirrhotic critically ill patients.

The outcome of patients with liver cirrhosis admitted to ICUs has been previously regarded as poor but have improved over ten years, especially for patients hospitalized for septic shock [15], a recent meta-analysis including 2523 cirrhotic patients reported a $42.7 \%$ ICU mortality rate [4]. However, it is unclear when this supportive care becomes futile, particularly in patients who are not candidates for liver transplant.

Reevaluation of SOFA score at day 3 or its variation between admission and day 3 ( $\triangle \mathrm{SOFA}$ ) are frequently used in critically ill cirrhotic patients to decide whether or not to maintain life-sustaining therapy $[4,5,16]$. CLIFSOFA and CLIF-C ACLF scores have also been used to evaluate patients' outcomes with cirrhosis $[17,18]$ and are known to have similar performance as the SOFA score in predicting short-term mortality [19]. Our study confirmed the prognostic ability of $\triangle$ SOFA for 28 -day mortality, did not find a significant improvement of death prediction by chronic liver failure scores and demonstrates that combining $\triangle N L R$ to $\triangle S O F A$ would improve 28 -day mortality prediction.

NLR has been studied in various conditions such as cardiovascular disease [20], renal failure [21] and acute pancreatitis [22]. NLR was also reported as a prognostic marker in unselected critically ill patients [7]. The cause responsible for NLR elevation correlating with poor outcome remains unclear. One of the most convincing hypothesis is that it may reflect both the rise in neutrophil count and the lymphocyte apoptosis induced by the overwhelming response to critical illness-related stress [7,23]. The compensatory anti-inflammatory response syndrome has evolved to serve as a counter-regulatory mechanism in the face of systemic inflammation. It represents a form of secondary immune deficiency state that can profoundly affect both innate and adaptive immune function and morbi-mortality [24]. Perhaps the most readily available marker of adaptive immune function in critical illness is the absolute lymphocyte count, which has been associated with mortality and secondary infection risk, especially in adults and children with sepsis [25].

Regarding cirrhotic patients, NLR analysis has emerged recently as a predictor of mortality. A cirrhosis-associated immune dysfunction associating features of chronic systemic inflammation (such as high neutrophil count) and immunodepression (such as lymphopenia) has been described $[17,26,27]$ and could be partly related to bacterial translocation and endotoxinemia due to the severity of the underlying liver disease $[17,28]$. This defective regulation of the inflammatory response has been associated with the development of organ failures and the risk of death $[17,26,29,30]$. Then, the NLR may be of particular interest and has been studied in different subpopulations of cirrhotic patients. For example, NLR is known to predict mortality in patients with stable liver cirrhosis [8] or in patients listed for liver transplantation [9] and has been analyzed as a marker of the disease progression [31,32].

However, to date, only one small study investigated the interest of NLR in the subpopulation of critically ill cirrhotic patients hospitalized for ACLF and suggested an association between the highest NLR value and 90-day mortality [33].

Our study provides additional data on the interest of NLR in critically ill cirrhotic population. First, we selected all cirrhotic patients still alive at day 3 . This timeframe requires special attention because ethical discussions about the intensity of care to provide usually arise at this time after a few days of optimal care before reevaluating the prognosis $[5,16,34]$. Our study is the first to propose 
(a)

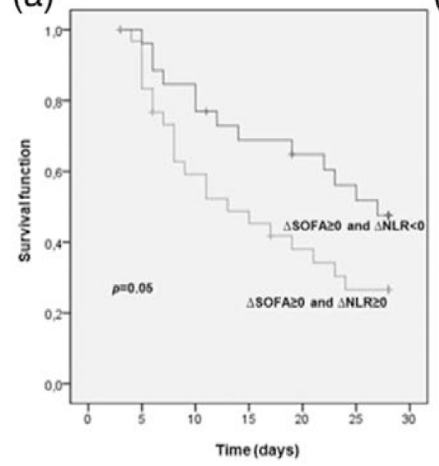

Test cohort

(b)

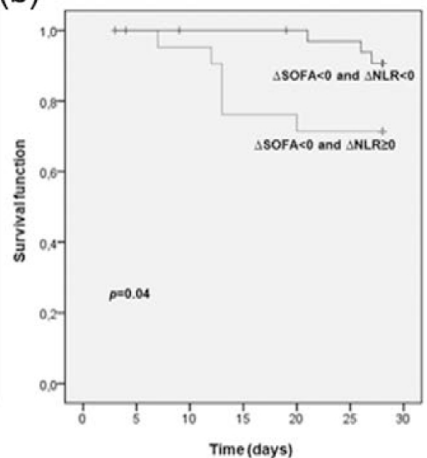

Validation cohort

(c)

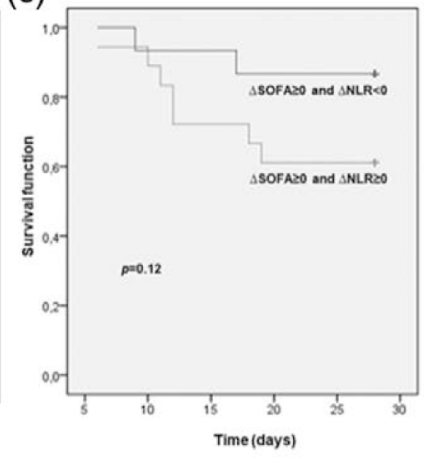

(d)

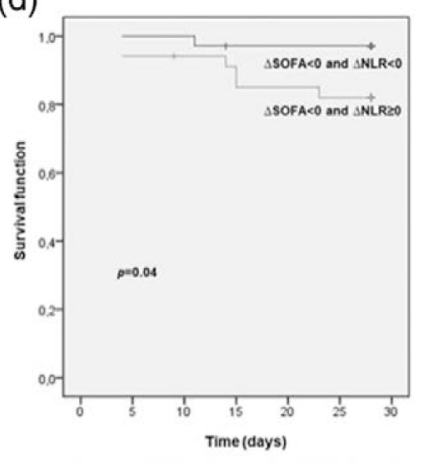

Fig. 3. Kaplan-Meier survival analysis according to the improvement of $\triangle N L R$ among patients that (1) increase SOFA score (a,c) (2) decrease SOFA score $(\mathrm{b}, \mathrm{d})$ between $\mathrm{DO}$ and D3. $\triangle \mathrm{SOFA} \geq 0$ and $\triangle N L R \geq 0$ indicate absence of improvement $(=0)$ or impairment of the value $(>0)$. $\triangle \mathrm{SOFA}<0$ and $\triangle N L R<0$ indicate improvement of the value between D0 and D3. NLR, neutrophil-to-lymphocyte ratio; SOFA, sequential organ failure assessment.

to integrate the NLR into the ethical discussion regarding therapies to maintain or not in ICU.

Second, instead of analyzing static data, we analyzed concomitant evolutions of NLR and usual prognostic scores during the first 3 days of hospitalization. De Grooth et al. [10] have indeed shown in a metaregression analysis the interest of using dynamic rather than static data for mortality prediction, especially with regard to the SOFA score. Moreover, a recent study published in 2018 by Karvellas et al. [35] highlighted the interest of a dynamic prognostication in critically ill cirrhotic patients.

Third, the goal of our study was not to replace usual organ failure-based scores but rather to analyze the additive value of $\triangle$ NLR to reinforce their predictive ability. Although some organ failure scores (like SOFA and CLIFSOFA) are interesting to predict patient mortality [4], they require a threshold to be determined and are probably not sufficient to identify patients for whom the continuation of intensive care therapies is futile and those with a better prognosis [36]. In addition, NLR's interest lies in the fact that it is very simple to obtain and easy to use biomarker. Therefore, we provide a pragmatic approach for clinicians which makes it easy to identify three groups of patients for the prognostication: those with a very good prognosis for whom both SOFA and NLR decrease; those with a poorer prognosis for whom both SOFA and NLR increase; and those who are in a grey zone of analysis in whom one score increases whereas the other decreases. Of note, it may have been interesting to investigate combined NLR and SOFA prognostic values in patients with different clinical courses of acute decompensation of cirrhosis, as described in the recent PREDICT study [11]. Indeed, NLR may capture the progression of systemic inflammation that was shown by Trebicka et al. [11] as a major pathogenic mechanism underlying the different patterns of cirrhosis progression.

Finally, we confirmed our results by an external validation cohort of 101 patients. Patients included in this validation cohort appear to be less severe than those in the test cohort. We were not able to control for these differences, but main results were confirmed in the validation cohort, suggesting the reproducibility of our results in various populations of critically ill cirrhotic patients.

This study has some limitations. First, its retrospective design may have biased the results and a prospective validation would further strengthen our results. Second, patients' screening and inclusion were based on coding data. It is, therefore, possible that some cirrhotic patients hospitalized during the study period could not be included because of coding inaccuracy. Indeed, only 116 ICU cirrhotic patients were recruited over a 7 -year period in the test cohort. However, this low rate may also reflect the current practice of many general and non-liver transplant hospitals, such the center in which our test cohort was recruited. Third, although results reach the significance threshold, $\triangle$ NLR does not perfectly discriminate patients at high risk of death, even in association with $\triangle \mathrm{SOFA}$, and can only be considered as a medical decision aid. For instance, the worst scenario combining increased $\triangle \mathrm{NLR}$ and $\triangle$ SOFA was still associated with 27 and $60 \%$ survival in the test and validation cohorts, respectively. Fourth, the NLR value and its evolution may have been influenced in case of sepsis, which account for a large part of the population of the test cohort.

\section{Conclusion}

$\triangle N L R$ is a predictor of mortality in critically ill cirrhotic patients still hospitalized in ICU after 3 days of full organ support. It could be used in association with $\triangle \mathrm{SOFA}$ as a prognostic biomarker that may help clinician decision-making in those patients with high ICU-death rates. Future studies are needed to consider the role of $\triangle$ NLR in the best management strategy of critically ill cirrhotic patients' which may include palliative care.

\section{Acknowledgements}

We are grateful to Dr. Jonathan Levy for his language help and to Dr. Anaïs Codorniu for her help in statistical analyzes.

This study was approved by the ethical committee of the French Society of Anesthesia and Intensive Care Medicine (NIRB 00010254-2019-033).

The datasets used and/or analyzed during the current study are available from the corresponding author on reasonable request.

M.G. and E.W. conceptualized the study, acquired the data, performed the statistical analysis, interpreted the data and drafted the manuscript. G.C. acquired the data, prepared and critically reviewed the manuscript. C.L. 
acquired the data. A.D.J., S.G., R.M., G.P. and S.J. prepared and critically reviewed the manuscript. C.P.B. conceptualized the study and prepared and critically reviewed the manuscript. F.T. conceptualized the study, interpreted the data and reviewed the manuscript. All authors read and approved the final manuscript.

\section{Conflicts of interest}

There are no conflicts of interest.

\section{References}

Majumdar A, Bailey M, Kemp WM, Bellomo R, Roberts SK, Pilcher D. Declining mortality in critically ill patients with cirrhosis in Australia and New Zealand between 2000 and 2015. I Hepatol 2017; 67:1185-1193.

2 Galbois A, Aegerter P, Martel-Samb P, Housset C, Thabut D, Offenstadt G, et al.; Collège des Utilisateurs des Bases des données en Réanimation (CUB-Réa) Group. Improved prognosis of septic shock in patients with cirrhosis: a multicenter study. Crit Care Med 2014; 42:1666-1675.

3 Piton G, Chaignat C, Giabicani M, Cervoni JP, Tamion F, Weiss E, et al. Prognosis of cirrhotic patients admitted to the general ICU. Ann Intensive Care 2016; 6:94.

4 Weil D, Levesque E, McPhail M, Cavallazzi R, Theocharidou E, Cholongitas E, et al.; METAREACIR Group. Prognosis of cirrhotic patients admitted to intensive care unit: a meta-analysis. Ann Intensive Care 2017; 7:33

5 Das V, Boelle PY, Galbois A, Guidet B, Maury E, Carbonell N, et al. Cirrhotic patients in the medical intensive care unit: early prognosis and long-term survival. Crit Care Med 2010; 38:2108-2116.

6 Ferreira FL, Bota DP, Bross A, Mélot C, Vincent JL. Serial evaluation of the SOFA score to predict outcome in critically ill patients. JAMA 2001; 286:1754-1758.

7 Salciccioli JD, Marshall DC, Pimentel MA, Santos MD, Pollard T, Celi LA, Shalhoub J. The association between the neutrophil-to-lymphocyte ratio and mortality in critical illness: an observational cohort study. Crit Care 2015; 19:13.

8 Kalra A, Wedd JP, Bambha KM, Gralla J, Golden-Mason L, Collins C, et al. Neutrophil-to-lymphocyte ratio correlates with proinflammatory neutrophils and predicts death in low model for end-stage liver disease patients with cirrhosis. Liver Transpl 2017; 23:155-165.

9 Leithead JA, Rajoriya N, Gunson BK, Ferguson JW. Neutrophil-tolymphocyte ratio predicts mortality in patients listed for liver transplantation. Liver Int 2015; 35:502-509.

10 de Grooth HJ, Parienti JJ, Oudemans-van Straaten HM. Should we rely on trials with disease- rather than patient-oriented endpoints? Intensive Care Med 2018; 44:464-466.

11 Trebicka J, Fernandez J, Papp M, Caraceni P, Laleman W, Gambino C, et al.; PREDICT STUDY group of the EASL-CLIF Consortium. The PREDICT study uncovers three clinical courses of acutely decompensated cirrhosis that have distinct pathophysiology. J Hepatol 2020; 73:842-854.

12 Pencina MJ, D'Agostino RB Sr, D’Agostino RB Jr, Vasan RS. Evaluating the added predictive ability of a new marker: from area under the ROC curve to reclassification and beyond. Stat Med 2008; 27:157-172.

13 Pencina MJ, Neely B, Steyerberg EW. RE: net risk reclassification P values: valid or misleading? J Natl Cancer Inst 2015; 107:355.

14 Pencina MJ, D'Agostino RB, Pencina KM, Janssens AC, Greenland $P$. Interpreting incremental value of markers added to risk prediction models. Am J Epidemiol 2012; 176:473-481.

15 Sauneuf B, Champigneulle B, Soummer A, Mongardon N, Charpentier J, Cariou A, et al. Increased survival of cirrhotic patients with septic shock. Crit Care 2013; 17:R78.

16 Cholongitas E, Betrosian A, Senzolo M, Shaw S, Patch D, Manousou $P$, et al. Prognostic models in cirrhotics admitted to intensive care units better predict outcome when assessed at $48 \mathrm{~h}$ after admission. $J$ Gastroenterol Hepatol 2008; 23:1223-1227.

17 Moreau R, Jalan R, Gines P, Pavesi M, Angeli P, Cordoba J, et al.; CANONIC Study Investigators of the EASL-CLIF Consortium.
Acute-on-chronic liver failure is a distinct syndrome that develops in patients with acute decompensation of cirrhosis. Gastroenterology 2013; 144:1426-1437, 1437.e1

18 Engelmann C, Thomsen KL, Zakeri N, Sheikh M, Agarwal B, Jalan R, Mookerjee RP. Validation of CLIF-C ACLF score to define a threshold for futility of intensive care support for patients with acute-on-chronic liver failure. Crit Care 2018; 22:254.

19 McPhail MJ, Shawcross DL, Abeles RD, Chang A, Patel V, Lee GH, et al. Increased survival for patients with cirrhosis and organ failure in liver intensive care and validation of the chronic liver failure-sequential organ failure scoring system. Clin Gastroenterol Hepatol 2015; 13:1353-1360.e8.

20 Bhat T, Teli S, Rijal J, Bhat H, Raza M, Khoueiry G, et al. Neutrophil to lymphocyte ratio and cardiovascular diseases: a review. Expert Rev Cardiovasc Ther 2013; 11:55-59.

21 Reddan DN, Klassen PS, Szczech LA, Coladonato JA, O'Shea S, Owen WF Jr, Lowrie EG. White blood cells as a novel mortality predictor in haemodialysis patients. Nephrol Dial Transplant 2003; 18:1167-1173.

22 Jeon TJ, Park JY. Clinical significance of the neutrophil-lymphocyte ratio as an early predictive marker for adverse outcomes in patients with acute pancreatitis. World J Gastroenterol 2017; 23:3883-3889.

23 Zahorec R. Ratio of neutrophil to lymphocyte counts-rapid and simple parameter of systemic inflammation and stress in critically ill. Bratis/ Lek Listy 2001; 102:5-14.

24 Greathouse KC, Hall MW. Critical illness-induced immune suppression: current state of the science. Am J Crit Care 2016; 25:85-92.

25 Hotchkiss RS, Tinsley KW, Swanson PE, Schmieg RE Jr, Hui JJ, Chang KC, et al. Sepsis-induced apoptosis causes progressive profound depletion of B and CD4+ T lymphocytes in humans. J Immunol 2001; 166:6952-6963.

26 Gandoura S, Weiss E, Rautou PE, Fasseu M, Gustot T, Lemoine F, et al. Gene- and exon-expression profiling reveals an extensive LPSinduced response in immune cells in patients with cirrhosis. $J$ Hepatol 2013; 58:936-948.

27 Weiss E, Rautou PE, Fasseu M, Giabicani M, de Chambrun M, Wan J, et al. Type I interferon signaling in systemic immune cells from patients with alcoholic cirrhosis and its association with outcome. J Hepatol 2017; 66:930-941.

28 Albillos A, de la Hera A, González M, Moya JL, Calleja JL, Monserrat J, et al. Increased lipopolysaccharide binding protein in cirrhotic patients with marked immune and hemodynamic derangement. Hepatology 2003; 37:208-217.

29 Thabut D, Massard J, Gangloff A, Carbonell N, Francoz C, NguyenKhac $\mathrm{E}$, et al. Model for end-stage liver disease score and systemic inflammatory response are major prognostic factors in patients with cirrhosis and acute functional renal failure. Hepatology 2007; 46:1872-1882.

30 Moreau R. The pathogenesis of ACLF: the inflammatory response and immune function. Semin Liver Dis 2016; 36:133-140.

31 Khoury T, Mari A, Nseir W, Kadah A, Sbeit W, Mahamid M. Neutrophilto-lymphocyte ratio is independently associated with inflammatory activity and fibrosis grade in nonalcoholic fatty liver disease. Eur J Gastroenterol Hepatol 2019; 31:1110-1115.

32 Ozturk K, Altun B, Kurt O, Demirci H. Neutrophil-to-lymphocyte ratio for predicting fibrosis in nonalcoholic fatty liver disease. Eur $J$ Gastroenterol Hepatol 2015; 27:1479.

33 Moreau N, Wittebole X, Fleury Y, Forget P, Laterre PF, CastanaresZapatero D. Neutrophil-to-lymphocyte ratio predicts death in acuteon-chronic liver failure patients admitted to the intensive care unit: a retrospective cohort study. Shock 2018; 49:385-392.

34 Gustot T, Fernandez J, Garcia E, Morando F, Caraceni P, Alessandria C, et al.; CANONIC Study Investigators of the EASL-CLIF Consortium. Clinical course of acute-on-chronic liver failure syndrome and effects on prognosis. Hepatology 2015; 62:243-252.

35 Karvellas CJ, Garcia-Lopez E, Fernandez J, Saliba F, Sy E, Jalan R, et al.; Chronic Liver Failure Consortium and European Foundation for the Study of Chronic Liver Failure. Dynamic prognostication in critically ill cirrhotic patients with multiorgan failure in ICUs in Europe and North America: a multicenter analysis. Crit Care Med 2018; 46:17831791.

36 Ginès P, Fernández J, Durand F. Management of critically-ill cirrhotic patients. J Hepatol 2012; 56 (Suppl 1):S13-S24. 